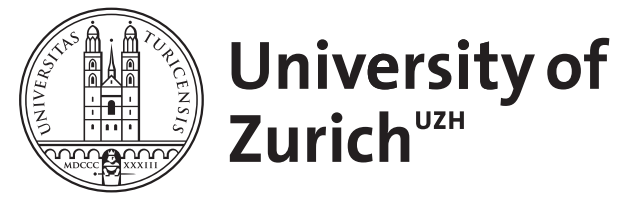

Zurich Open Repository and Archive

University of Zurich

University Library

Strickhofstrasse 39

CH-8057 Zurich

www.zora.uzh.ch

Year: 2018

\title{
Ultramarathon mit Diabetes mellitus Typ 1
}

Knechtle, Beat ; Nikolaidis, Pantelis T

DOI: https://doi.org/10.1024/1661-8157/a002999

Posted at the Zurich Open Repository and Archive, University of Zurich

ZORA URL: https://doi.org/10.5167/uzh-152314

Journal Article

Accepted Version

Originally published at:

Knechtle, Beat; Nikolaidis, Pantelis T (2018). Ultramarathon mit Diabetes mellitus Typ 1. Praxis, 107(14):777-781. DOI: https://doi.org/10.1024/1661-8157/a002999 
1 Medbase St. Gallen Am Vadianplatz, St. Gallen ${ }^{1}$

2 Exercise Physiology Laboratory, Nikaia, Griechenland ${ }^{2}$

3

4 Beat Knechtle ${ }^{1}$, Pantelis T. Nikolaidis ${ }^{2}$

5

6 Ultramarathon mit Diabetes mellitus Typ 1

7

$8 \quad$ Ultra-marathon running with diabetes

9

10

11

12

13

14

15

16

17

18 


\section{Im Artikel verwendete Abkürzungen}

20

21

BMI

Body Mass Index

22

CGM

Continuous Glucose Monitoring

23

$\mathrm{HbA}_{1 \mathrm{c}}$

Hämoglobin $A_{1 c}$

24

I.E.

Internationale Einheiten

25

Kcal

Kilokalorie

26

KHE

Kohlenhydrateinheiten-Faktor

27

28

29

30

31

32

33

34

35

36

37

38

39

40

41

42

43

44 


\section{Einleitung}

Ein Ultramarathon bezeichnet eine Laufstrecke die länger ist als eine klassische Marathonstrecke $(42.195 \mathrm{~km})$ resp. eine Ausdauerbelastung die über 6 Stunden und länger dauert. Generell wird bei den Ultramarathons unterschieden in Läufe die über die Strecke definiert sind (in Kilometer oder in Meilen) [1] oder in Läufe die über die Zeit (6 Stunden, 12 Stunden, 24 Stunden, bis 10 Tage) begrenzt werden [2].

In den letzten Jahrzehnten hat sich im Ultramarathonbereich die Zahl der Wettkämpfe deutlich erhöht [3] und die Zahl der erfolgreichen Finisher teils exponentiell entwickelt [4]. Besonders populär sind im Ultramarathon-Bereich die 100-km Läufe [5], die 100-Meilen Läufe [6], die Läufe in Stunden resp. Tagen von 6 Stunden bis 10 Tagen [2] und Mehretappenläufe wie ein Lauf durch eine Wüste [7], durch ein Land [8] oder durch einen ganzen Kontinent [9].

Ultraläufer unterscheiden sich von der allgemeinen Bevölkerung relativ deutlich. In der Regel sind Ultraläufer gesunde Menschen im mittleren Alter zwischen 30 und 40 Jahren mit einem sehr hohen Trainingsaufwand und langjähriger Erfahrung im Marathon- und Ultramarathon-Laufen [10,11]. Die Prävalenz für chronische Erkrankungen wie etwa eine koronare Herzkrankheit oder ein Diabetes mellitus liegt bei Ultraläufern bei deutlich unter $1 \%$ [10].

In den letzten Jahren gab es allerdings ganz vereinzelte Berichte dass auch Ausdauersportler mit insulinpflichtigem Diabetes mellitus erfolgreich Ultrawettkämpfe erfolgreich absolvieren können [12]. In einer grösseren Studie wurden Triathleten mit insulinpflichtigem Diabetes mellitus beschrieben die einen Ironman-Triathlon erfolgreich absolviert haben [13]. Sogar deutlich längere Strecken sind möglich. Aus Fallberichten ist bekannt dass ein Triathlet mit insulinpflichtigem Diabetes mellitus 
einen Double Iron Ultratriathlon (7.6 km Schwimmen, 360 km Radfahren und 84.4 km Laufen) gefinisht hat [14] sowie ein Triathlet mit insulinpflichtigem Diabetes mellitus der einen Ultraman-Triathlon (Strecke eines Double Iron aufgeteilt auf 3 Tagesetappen) erfolgreich absolviert hat [15].

Im Bereich des Laufsports gibt es Feldstudien mit insulinpflichtigen Diabetikern die erfolgreich Ausdauerläufe wie einen Halbmarathon [16], einen Marathon [17,18] oder in einzelnen Fällen gar einen Ultramarathon [19] erfolgreich absolviert haben.

Wir wissen aus der Literatur dass Sportler mit insulinpflichtigem Diabetes mellitus lange Laufwettbewerbe bis hin zu Ultramarathons schaffen, hingegen wissen wir nichts über den Langzeitverlauf und das Training dieser Ausdauersportler. Speziell kennen wir den Zusammenhang zwischen Training und Zuckerstoffwechsel $\left(\mathrm{HbA}_{1} \mathrm{c}\right)$ über die Jahre nicht.

Im vorliegenden Fallbericht berichten wir über einen jetzt 62-jährigen Läufer der seit seinem 21. Lebensjahr einen juvenilen Diabetes mellitus Typ 1 hat und seit seinem 32. Lebensjahr Marathons und seit seinem 34. Lebensjahr Ultramarathons läuft. Anhand seiner Aufzeichnungen des Trainings, des $\mathrm{HbA}_{1} \mathrm{c}$ und der Wettkampfresultate über einen Zeitraum von mehreren Jahrzehnten können wir zeigen dass man auch mit einem insulinpflichtigen Diabetes mellitus Typ I erfolgreich über Jahrzehnte Marathons und Ultramarathons bestreiten kann. Insbesondere können wir einen Einblick zum Zusammenhang zwischen Training und den Verlauf des $\mathrm{HbA}_{1} \mathrm{c}$ über die Jahre gewinnen. Diese Resultate sind für Hausärzte und andere Sportler mit einem insulinpflichtigen Diabetes mellitus Typ I von Interesse für die Planung von Training und Wettkampf. 


\section{Resultate}

Wir beschreiben in einem ersten Abschnitt den Läufer mit seinem Diabetes und dann in einem zweiten Abschnitt seine sportlichen Leistungen. Wir versuchen dann Zusammenhänge zwischen Angaben des Läufers $\left(\mathrm{HbA}_{1 c}\right.$, Training) und seinen Laufleistungen zu finden.

\section{Der Läufer, sein Diabetes und sein Training}

Unser Läufer, Jahrgang 1955, wird jetzt 63 Jahre alt. Im Jahre 1976 - im Alter von 21 Jahren - wurde bei inm Diabetes mellitus Typ 1 diagnostiziert. Im Jahre 1984 - im Alter von 29 Jahren -, begann er mit Laufen. Damals lief er 2-3 Einheiten pro Woche und jeweils 2-3 km pro Einheit. Mit 32 Jahren lief er seinen ersten Marathon und mit 34 Jahren den ersten Ultramarathon.

Im Alter von 16 Jahren wog er 133 kg, im Laufe der Zeit konnte er das Gewicht auf 110-115 kg reduzieren. Als der Diabetes mellitus nachgewiesen wurde senkte er das Gewicht auf 85-90 kg, in den letzten Jahren und Jahrzenten wog er um 80-85 kg.

Da der Diabetes-Typ recht ungewöhnlich erscheint und der Läufer mit 16 Jahren extrem adipös war wurde 2011 der C-Peptid-Wert überprüft. Bei einem Wert von $17.0 \mathrm{pmol} / / \mathrm{mit}$ einem Normwert von 364-1655 pmol// geht klar hervor dass es sich nicht um einen Diabetes mellitus Typ 2 handelt.

Als grundsätzliches Ernährungsschema ernährt er sich im Alltag vollwertig nach Kollath/Bruker mit nur zwei Mahlzeiten am Tag ohne Zwischenmahlzeiten. Am Morgen nimmt er einen Frischkornbrei aus $40 \mathrm{~g}$ Getreide mit etwa $300 \mathrm{~g}$ Obst und ein wenig Sahne die mit Süssstoff versetzt ist. Am Abend isst er frische Salate, Käse, Gemüse und Vollkornbrot mit Butter, zum Dessert noch Äpfel. In der Regel kommt er so auf etwa 30 KHE pro Tag, entsprechend etwa $300 \mathrm{~g}$ Kohlenhydraten pro Tag. 
119 Für den Verlauf seines Diabetes hat der Läufer das $\mathrm{HbA}_{1 c}$ seit 2001 kontinuierlich

120 protokolliert. Im Laufe der Jahre fiel der Wert kontinuierlich ab (Abbildung 1).

121 Sein Trainingsvolumen hat er seit 1984 minutiös protokolliert mit den monatlichen

122 Laufkilometern. Das Training betreibt er nicht aus einem Zwang heraus sondern für

123 sein persönliches Wohlbefinden. Ein eigentliches spezifisches Lauftraining betreibt er

124 nicht, sondern baut das Laufen im Sinne eines Walking in den Alltag ein. Das

125 Lauftraining folgt auch nicht einem sturen Trainingsplan. Als Lauf- resp.

126 Wandertraining betreibt er viele Touren im bergigen Gelände mit 500 bis 1000

127 Höhenmeter. Bei diesen Bergtouren mit bis zu 2500 Höhenmetern baut er auch

128 schnelle Einheiten ein. Die Hochgebirgstouren mit bis zu insgesamt 2500

129 Höhenmeter bergauf evtl. auch wieder bergab macht er nur im Sommer bei guten

130 Wetterbedingungen, dabei steht im Vordergrund das Ziel den geplanten Gipfel zu

131 erreichen. Das Lauftempo ist bei den zum Teil schwierigen Wegen und evtl. auch

132 großer Hitze nicht so entscheidend.

133 Es zeigt sich eine signifikante Zunahme des Laufvolumens im Laufe der Jahrzehnte

134 (Abbildung 2). Wenn wir noch einen Zusammenhang zwischen dem $\mathrm{HbA}_{1 \mathrm{c}}$ und dem

135 Laufvolumen der drei Monaten vor der jeweiligen Messung des $\mathrm{HbA}_{1 \mathrm{c}}$ herstellen, so

136 zeigt sich ein klarer Zusammenhang zwischen Laufvolumen und $\mathrm{HbA}_{1 \mathrm{c}}$ in dem Sinne

137 dass das mit Zunahme des Laufvolumens über die Jahre das $\mathrm{HbA}_{1 \mathrm{c}}$ kontinuierlich

138 abgenommen hat (Abbildung 3).

\section{Die Wettkämpfe}

140 Wir teilen seine bisherigen Laufresultate in distanz-limitiere (Marathon, 100km) und

141 zeit-limitiere (6 Stunden, 12 Stunden, 24 Stunden, 48 Stunden, 72 Stunden, 6 Tage)

142 Läufe ein. Zwischen 1987 und 2004 lief er 15 Marathons mit einer mittleren

143 Schlusszeit von 4:22:33 h:min:s und zwischen 1989 und 200511 100-km

144 Ultramarathons mit einer mittleren Schlusszeit von 17:02:00 h:min:s (Tabelle 1). Sein 
145 Hauptaugenmerk liegt bei den Zeit-limitierten Läufen. Bei diesen Läufen (Tabelle 2)

146 absolvierte er 1998 und 1999 je einen 6-Stunden-Lauf mit einer mittleren erzielten

147 Strecke von $51.7 \mathrm{~km}$. Von 1991 bis 1995 lief er zwei 12-Stunden-Läufe mit einer

148 mittleren Strecke von 80.4 km. Bei den 24-Stunden-Läufen kam er von 1994 bis

1492017 auf total 48 Wettkämpfe wobei die Leistung im Schnitt bei rund $133 \mathrm{~km}$ lag und

150 über die Jahre ohne nachweisbare Veränderung lag (Abbildung 4). Über 48 Stunden

151 machte er zwischen 1999 und 2005 total 6 Wettkämpfe mit einer mittleren Leistung

152 von $184.8 \mathrm{~km}$. Während er über 72 Stunden nur einen Wettkampf mit $288.9 \mathrm{~km}$ lief

153 absolvierte er zwischen 2008 und 2016 drei 6-Tage-Läufe mit einer mittleren

154 Leistung von $438 \mathrm{~km}$.

155 Wir fokussieren uns auf die 24-Stunden-Läufe da sich diese Wettkampfform zu

156 seiner Spezialdisziplin entwickelt hat. Bei den 24-Stunden-Läufen hat er ein klares

157 Ernährungsschema. Der Start bei diesen Wettkämpfen ist in der Regel am Mittag um

15812 Uhr. Vom offiziellen Verpflegungsstand der Veranstalter nimmt er je nach

159 Blutzucker Kuchenstücke oder Salzstangen und trinkt dazu konventionelles Coca

160 Cola $^{\circledR}$. Nach rund 9 Stunden Laufdauer (am Abend um 21 Uhr) nimmt er ein

161 Abendessen bestehend aus Kartoffeln und Gemüse (ca. 5 KHE) zu sich. Nach rund

16214 Stunden (am frühen Morgen um 2 Uhr) nimmt er Kaffee und Kuchen (ca. 5 KHE).

163 Die Mengen sind immer in etwa gleich bei den verschiedenen Wettkämpfen.

164 Das Prinzip der Blutzuckermessung

165 Für die Blutzuckerkontrollen verwendete er bis 2015 das Accu-Check Mobile

166 Testgerät für die regelmässigen Blutzuckerkontrollen (www.accu-chek.ch). Damit

167 konnte er auch gut während eines Laufs den Blutzucker messen ohne stehen bleiben

168 zu müssen. Er trug das Messgerät sowie die Stechhilfe in einem Brustbeutel, stach

169 sich dann in den Finger, verstaute die Stechhilfe wieder im Beutel, nahm das

170 Messgerät heraus, trug das Blut auf und führte die Messung durch. Seit September 
1712015 läuft die Messung über CGM (Continuous Glucose Monitoring) mit Medtronic

172 MiniMed 640G (www.medtronic-diabetes.ch) und Enlite Glucosesensor.

173 Die Insulinabgabe erfolgte bis 2003 in konventioneller Form, seit 2004 problemlos mit 174 der Insulinpumpe, von 2004 bis 2009 mit RocheD-tron, von 2009 bis 2012 mit Roche 175 Spirit, von 2013 bis 2015 mit MedtronicMiniMed 640G und ab 2015 mit CGM

176 (continuous glucose monitoring) (www.medtronic-diabetes.ch/de/minimed-

177 produkte/minimed-640g-insulinpumpe). Mit der klassischen Insulinapplikation hat er

178 sich immer vor dem Essen das Insulin gespritzt, je nach Situation hat er das auch 179 während dem Laufen gemacht.

180 Die Hypoabschaltung der $640 \mathrm{G}$ ist für den Läufer extrem nützlich. Während des

181 Trainings ist die Hypoabschaltung sehr häufig aktiv mit dem Vorteil dass er sich auf

182 das Laufen konzentrieren kann und den Blutzuckerwert wesentlich weniger

183 kontrollieren muss. Sobald der Blutzuckerwert absinkt stellt auch die Pumpe ab und

184 die Gefahr einer Hypoglykämie ist wesentlich geringer. Bei einem steigenden

185 Blutzuckerwert stellt die Pumpe wieder an und so bleibt der Blutzuckerwert fast

186 immer in einem Bereich von $3.5-7 \mathrm{mmol} / \mathrm{l}$. Dies ist besonders nützlich im Winter bei

187 Kälte, Schnee und Regen, denn da bleiben die Hände besser in den dicken und

188 warmen Handschuhen als die Pumpe aus der Hosentasche zu ziehen, den Wert

189 abzulesen und die Pumpe abzustellen.

190 Der tägliche Insulinbedarf liegt zurzeit bei etwa 35-45 Einheiten wenn er eine übliche

191 Ernährung hat. Allerdings variiert der tägliche Insulinbedarf was er auf die

192 unterschiedliche Resorption des Insulins zurückführt.

193 Solange er kein CGM hatte reduzierte er die Basalrate der Pumpe um 70\% während 194 eines Laufes, sofern die unter Belastung gemessenen Blutzuckerwerte in der Norm 195 lagen. Falls ein Korrekturbolus nötig wurde hatte er kein festes Schema sondern nach eigener Erfahrung dosiert. Es traten dabei keine Hypoglykämien auf. 
197 Auch die sehr seltenen Blutzuckerentgleisungen wären eher typisch für einen

198 Diabetes mellitus Typ 2. Der Läufer kann aufgrund seiner Erfahrung das

199 Blutzuckerverhalten sehr gut einschätzen. Er kann problemlos 10 und mehr Stunden

200 Laufen ohne etwas zu Essen und verliert dabei seine Leistungsfähigkeit nicht.

201 Dadurch kann er mit sehr wenig Insulin auskommen und es gibt keine Probleme

202 durch einen zu hohen oder auch zu niedrigen Bolus. Dabei ist das CGM sehr hilfreich

203 weil er bereits in einem sehr frühen Stadium erkennen kann dass die

204 Hypoabschaltung nicht ausreicht und er bereits mit einem kleinen Schluck Coca

205 Cola $^{\circledR}$ die Werte anpassen kann. Genauso ist es bei einem ansteigenden

206 Blutzuckerwert bei dem bereits 0.5 IE Insulin ausreichen können um den Wert im

207 optimalen Bereich zu halten. 


\section{Diskussion}

221 Bei der Analyse der Zahlen dieses sehr aussergewöhnlichen 62-jährigen Läufers mit

222 Diabetes mellitus Typ I seit dem 21. Lebensjahr fallen mehrere wichtige Punkte auf

223 wie (1) die sehr hohe Zahl von 48 absolvierten 24-Stunden-Läufe, (2) eine signifikante Zunahme des monatlichen Laufvolumens, (3) eine signifikante Abnahme des $\mathrm{HbA}_{1 \mathrm{c}}$ über die Jahre sowie (4) ein signifikanter Zusammenhang zwischen den monatlichen Laufkilometern und dem $\mathrm{HbA}_{1 \mathrm{c}}$.

\section{Anzahl absolvierter 24-Stunden-Läufe}

Unser Läufer hat von 1994 bis 2017 total 48 24-Stunden-Läufe absolviert, das gibt im Schnitt zwei solcher Läufe pro Jahr. Daneben hat er noch andere Läufe wie 100-kmLäufe und 6-Stunden-Läufe bis hin zu 6-Tages-Läufen gemacht. Eine Auswertung von 20’238 erfolgreichen Läufen von Frauen and 76‘888 erfolgreichen Läufen von Männern von Läufen über 6 Stunden bis hin zu 6 Tagen aus den Jahren 1975-2013 zeigte dass etwa $50 \%$ der Starter nur einen solchen Lauf je gemacht haben und nur 10\% 7 und mehr Läufe je im Leben gemacht haben [2]. Somit muss die Leistung dieses Läufers als absolut aussergewöhnlich bezeichnet werden, insbesondere da er noch mit einer chronischen Stoffwechselerkrankung ein Handicap hat.

\section{Abnahme des $\mathrm{HbA}_{1 \mathrm{c}}$ über die Jahre}

Wir fanden über die letzten fast 20 Jahre eine signifikante Abnahme des $\mathrm{HbA}_{1 c^{-}}$ Wertes über die Zeit sowie einen signifikanten Zusammenhang zwischen der Zunahme der monatlichen Laufkilometern und der Abnahme des $\mathrm{HbA}_{1 \mathrm{c}}$-Wertes. Wir könnten nun postulieren dass das gesteigerte Laufvolumen zu einer Verbesserung der diabetischen Stoffwechsellage geführt hat. Effektiv führt ein intensives Ausdauertraining bei Personen mit Diabetes mellitus Typ 2 zu einer Abnahme des $\mathrm{HbA}_{1 \mathrm{c}}$-Wertes [20]. 
Allerdings ist es auch möglich dass der gesunkene Wert des $\mathrm{HbA}_{1 \mathrm{c}}$-Wertes an einer Therapieanpassung liegt da der Läufer seit 2004 eine Insulinpumpe hat und seit 2015 ein CGM. Mit der Insulinpumpe dürfte eine Verbesserung der Einstellung des Diabetes möglich sein [21] und mit dem Gebrauch des CGM könnte noch eine weitere Verbesserung der Diabeteseinstellung möglich werden [22]. Bei Personen mit Diabetes mellitus Typ 1 sind das Management der Kontrolle der Blutglukose sowie eine mögliche Hypoglykämie die grössten Probleme für ein regelmässiges körperliches Training. Mit einer Insulinpumpe sowie dem CGM haben Personen mit einem Diabetes mellitus Typ 1 nun sehr gute Möglichkeiten ihren Blutzucker unter Belastung konstant zu halten [21].

Da der Läufer im Laufe der Jahre sein Gewicht reduziert hat könnte dies ein weiterer Grund für die Abnahme des $\mathrm{HbA}_{1 c}$-Wertes sein. Es ist nachgewiesen dass körperliches Training Marker des Schweregrads von Typ I-Diabetes mellitus nachweislich beeinflusst [23]. In Personen mit Diabetes mellitus Typ 2 führt eine Reduktion des Fettgewebes sowie eine Verbesserung der Fitness zu einer Abnahme des $\mathrm{HbA}_{1 \mathrm{c}}$-Wertes [24].

Das Gewicht kann aber auch aufgrund des Trainings gesunken sein, zumal der Läufer ja über die Jahre sein Laufvolumen gesteigert hat. Bei Ultraläufern ist bekannt dass sie mit zunehmendem Alter weniger an Gewicht zulegen als die allgemeine Bevölkerung [25].

\section{Konstante Wettkampfleistung und gesteigertes Trainingsvolumen}

Unser Läufer hat sein monatliches Laufvolumen im Laufe der letzten Jahrzehnte signifikant gesteigert. Wir haben Zahlen über 33 Jahre (1994-2017) die kaum vergleichbar sind mit Zahlen der Literatur. In einem Fallbericht wurde das Trainingsvolumen von einem Triathleten über eine halb so lange Zeit (17 Jahre, 1995-2012) für Schwimmen, Laufen und Radfahren zusammengestellt. Dieser 
271 Triathlet hat über all die Jahre keine Steigerung des Volumens im Radfahren und

272 Laufen zustande gebracht und sein Volumen im Schwimmen sogar noch reduziert

273 [26]. Auch aus trainingstechnischer Sicht muss die Leistung dieses Läufers als

274 absolut aussergewöhnlich bezeichnet werden.

275 Eine mögliche Erklärung für die Steigerung des Laufvolumens in den letzten Jahren

276 könnte die Teilnahme an den drei 6-Tage-Läufen in 2008, 2010 und 2016 sein. Es ist

277 belegt dass Ultraläufer im Vergleich zu Marathonläufern ein deutlich höheres

278 Trainingsvolumen haben [6]. Unser Läufer hat möglicherweise aus der Erfahrung der

279 vorangegangen 24-Stunden-Läufe sowie der 48-Stunden-Läufe von 1999-2005

280 gelernt dass er das Laufvolumen für einen 6-Tage-Lauf noch einmal deutlich steigern 281 muss.

\section{Zusammenhang zwischen Laufkilometern und $\mathrm{HbA}_{1 \mathrm{c}}$}

283 Wenn wir die $\mathrm{HbA}_{1 \mathrm{c}}$-Werte den Laufkilometern der drei letzten Monate vor der

Messung gegenüberstellen so finden wir einen inversen Zusammenhang im Sinne geführt hat. In Personen mit Diabetes mellitus Typ 2 zeigt sich das sich langjähriges körperliches Training günstig auf die Kontrolle des Blutzuckers auswirkt [27]. Nachweislich führt ein kontrolliertes und strukturiertes Training zu einer Abnahme des $\mathrm{HbA}_{1 \mathrm{c}}$-Wertes bei Patienten mit Diabetes mellitus Typ $2 \mathrm{im}$ Gegensatz zu einer unstrukturierten körperlichen Aktivität [28]. 
296 Key Messages

297 - Es ist möglich während über 20 Jahren jährlich mehr als einen 24-Stunden-

298 Lauf zu bestreiten und dabei keine Leistungsverschlechterung einzugehen

299 - Es ist möglich auch mit zunehmendem Alter das Laufvolumen als Läufer über

300 die Jahre noch zu steigern

301 - Es scheint dass eine Steigerung des Laufvolumens zu einer Reduktion des

$302 \quad \mathrm{HbA}_{1 \mathrm{c}}$-Wertes führt

303 - Personen mit insulinpflichtigem Diabetes mellitus Typ 1 können mit einer

304 Insulinpumpe und kontinuierlichem Glukose Monitoring (CGM) problemlos

305 auch sehr lange Ausdauerbelastungen bestreiten

306

307

308

309

310

311

312

313

314

315

316

317

318

319

320

321 
325 Wir berichten über einen 63-jährigen Läufer mit insulin-pflichtigem Diabetes mellitus

326 Typ 1 seit dem 21. Lebensjahr. Im Alter von 32 Jahren lief er den ersten Marathon,

327 mit 34 Jahren den ersten Ultramarathon. Bisher hat er über 90 Marathons und

328 Ultramarathons bestritten. Dank einer Insulinpumpe und kontinuierlichem Glukose

329 Monitoring hat er bisher 48 24-Stunden-Läufe mit einer durchschnittlichen Leistung

330 von $133 \mathrm{~km}$ bestritten. Die Analyse des Trainings sowie der $\mathrm{HbA}_{1 \mathrm{c}}$-Werte zeigt eine

331 signifikante Zunahme des monatlichen Laufvolumens, eine signifikante Abnahme des

$332 \mathrm{HbA}_{1 \mathrm{c}}$ über die Jahre sowie einen signifikanten Zusammenhang zwischen den

333 monatlichen Laufkilometern und dem $\mathrm{HbA}_{1 \mathrm{c}}$.

334 Schlüsselwörter

335 Ausdauerbelastung; Laufen; Stoffwechselstörung 
354 We report the case of a 63-year-old runner with type 1 diabetes mellitus requiring 355 insulin since the age of 21 years. At age of 32 years, he ran the first marathon, and at 356 the age of 34 years the first ultra-marathon. So far, he has finished more than 90 357 marathons and ultramarathons. Thanks to an insulin pump and continuous glucose 358 monitoring, he has so far completed 48 24-hour runs with an average distance of 133 $359 \mathrm{~km}$. The analysis of running volume and $\mathrm{HbA}_{1 \mathrm{c}}$ values showed a significant increase 360 in monthly exercise volume, a significant decrease in $\mathrm{HbA}_{1 \mathrm{c}}$ over the years, and a 361 significant correlation between monthly running kilometers and $\mathrm{HbA}_{1 \mathrm{c}}$.

\section{Key words}

363 Endurance performance, running, metabolic disorder 
380 Korrespondenzadresse

381 Prof. Dr. med. Beat Knechtle

382 Facharzt FMH für Allgemeinmedizin

383 Medbase St. Gallen Am Vadianplatz

384 Vadianstrasse 26

3859001 St. Gallen

386 Telefon $\quad+41$ (0) 712269300

387 Telefax +41 (0) 712269301

388 E-Mail beat.knechtle@hispeed.ch

389

390

391

392

393

394

395

396

397

398

399

400

401

402

403

404

405

406

407

408

409 


\section{Literaturverzeichnis}

411 1. Romer T, Rust CA, Zingg MA et al. Age and ultra-marathon performance - 50 to $1,000 \mathrm{~km}$ distances from 1969 - 2012. SpringerPlus 2014; 3: 693

2. Knechtle B, Valeri F, Zingg MA et al. What is the age for the fastest ultramarathon performance in time-limited races from $6 \mathrm{~h}$ to 10 days? Age

10. Hoffman MD, Krishnan E. Health and exercise-related medical issues among

8. Knechtle B, Duff B, Schulze I et al. Anthropometry and pre-race experience of finishers and nonfinishers in a multistage ultra-endurance run-deutschlandlauf 2007. Perceptual and Motor Skills 2009; 109: 105-118

9. Schütz UH, Schmidt-Trucksäss A, Knechtle B et al. The Transeurope Footrace Project: longitudinal data acquisition in a cluster randomized mobile $\mathrm{MRI}$ observational cohort study on 44 endurance runners at a 64-stage 4,486 $\mathrm{km}$ transcontinental ultramarathon. BMC medicine 2012; 10: 78 1,212 ultramarathon runners: Baseline findings from the Ultrarunners Longitudinal TRAcking (ULTRA) Study. PLoS ONE 2014; 9

11. Knechtle B. Ultramarathon runners: Nature or nurture? International Journal of Sports Physiology and Performance 2012; 7: 310-312

12. Ratjen I, Weber KS, Roden M et al. Type 1 Diabetes Mellitus and Exercise in Competitive Athletes. Experimental and Clinical Endocrinology and Diabetes 2015; 123: 419-422 
13. Boehncke S, Poettgen $\mathrm{K}$, Maser-Gluth $\mathrm{C}$ et al. [Endurance capabilities of triathlon competitors with type 1 diabetes mellitus]. Deutsche medizinische Wochenschrift (1946) 2009; 134: 677-682

14. Vlahek P, Car S, Ostroški I. Sweet $452 \mathrm{~km}$ - a report on the first type 1 diabetes patient to finish Double Ironman, a 30- hour endurance triathlon race. Croatian Medical Journal 2013; 54: 306-307

15. Bach CW, Baur DA, Hyder WS et al. Blood glucose kinetics and physiological changes in a type 1 diabetic finisher of the Ultraman triathlon: a case study. European Journal of Applied Physiology 2017; 117: 913-919

16. Murillo S, Brugnara L, Novials A. One year follow-up in a group of halfmarathon runners with type-1 diabetes treated with insulin analogues. Journal of Sports Medicine and Physical Fitness 2010; 50: 506-510

17. Cauza E, Hanusch-Enserer U, Strasser B et al. Continuous glucose monitoring in diabetic long distance runners. International Journal of Sports Medicine 2005; 26: 774-780

18. Knechtle B, Nikolaidis PT. Marathon running does not prevent diabetes.

19. Gawrecki A, Zozulinska-Ziolkiewicz D, Matejko B et al. Safe Completion of a Trail Running Ultramarathon by Four Men with Type 1 Diabetes. Diabetes technology \& therapeutics 2018, DOI: 10.1089/dia.2017.0296

20. Støa EM, Meling S, Nyhus LK et al. High-intensity aerobic interval training improves aerobic fitness and $\mathrm{HbA} 1 \mathrm{c}$ among persons diagnosed with type 2 diabetes. European Journal of Applied Physiology 2017; 117: 455-467

21. Codella R, Terruzzi I, Luzi L. Why should people with type 1 diabetes exercise regularly? Acta Diabetologica 2017; 54: 615-630

22. Kapitza C, Hövelmann U, Nosek L et al. Continuous glucose monitoring during exercise in patients with type 1 diabetes on continuous subcutaneous insulin infusion. Journal of Diabetes Science and Technology 2010; 4: 123131

23. Jewiss D, Ostman $\mathrm{C}$, King $\mathrm{N}$ et al. Clinical Outcomes to Exercise Training in Type 1 Diabetes: A Systematic Review and Meta-Analysis. Diabetes research and clinical practice 2017, DOI: 10.1016/j.diabres.2017.11.036

24. Sénéchal M, Swift DL, Johannsen NM et al. Changes in body fat distribution and fitness are associated with changes in hemoglobin $A<$ inf $>1 \mathrm{c}</$ inf $>$ after 9 months of exercise training: Results from the HART-D study. Diabetes Care 2013; 36: 2843-2849 
25. Hoffman MD, Chen L, Krishnan E. Body mass index and its correlates in 1,212 ultramarathon runners: Baseline findings from the ultra study. Journal of Physical Activity and Health 2014; 11: 1549-1555

26. Zingg MA, Pazahr S, Morsbach F et al. No damage of joint cartilage of the lower limbs in an ultra-endurance athlete--an MRI-study. BMC musculoskeletal disorders 2013; 14: 343

27. Najafipour F, Mobasseri M, Yavari A et al. Effect of regular exercise training on changes in $\mathrm{HbA1c}, \mathrm{BMI}$ and VO2max among patients with type 2 diabetes mellitus: an 8-year trial. BMJ open diabetes research \& care 2017; 5: e000414

28. Sanghani NB, Parchwani DN, Palandurkar KM et al. Impact of lifestyle modification on glycemic control in patients with type 2 diabetes mellitus. Indian journal of endocrinology and metabolism 2013; 17: 1030-1039 


\begin{tabular}{cc}
\hline \multicolumn{3}{c}{ Marathon } \\
\hline Jahr & Zeit (h:min:s) \\
\hline 1987 & $4: 47: 12$ \\
1988 & $3: 55: 29$ \\
1988 & $4: 16: 00$ \\
1988 & $4: 32: 20$ \\
1988 & $4: 30: 50$ \\
1988 & $4: 39: 10$ \\
1988 & $4: 02: 21$ \\
1989 & $4: 08: 30$ \\
1989 & $4: 12: 33$ \\
1990 & $3: 57: 15$ \\
1990 & $4: 17: 54$ \\
1990 & $4: 40: 12$ \\
1991 & $4: 23: 48$ \\
1992 & $4: 15: 38$ \\
2004 & $4: 59: 07$ \\
\hline $100-k m$ Ultra-Marathon \\
\hline $19 h r$ & Zeit (h:min:s) \\
\hline 1999 & $13: 47: 30$ \\
1991 & $15: 53: 40$ \\
1992 & $18: 53: 06$ \\
1993 & $16: 15: 55$ \\
1994 & $18: 50: 05$ \\
1995 & $15: 45: 57$ \\
1996 & $18: 46: 04$ \\
1997 & $16: 12: 58$ \\
1998 & $16: 51: 42$ \\
2005 & $17: 37: 07$ \\
& $18: 27: 55$ \\
\hline
\end{tabular}

511

514

515

516

517

518

519

520

521

Tabelle 1: Läufe aufgeteilt nach Strecke (Marathon und 100-km Ultramarathon) 


\begin{tabular}{|c|c|}
\hline Jahr & Leistung (km) \\
\hline \multicolumn{2}{|c|}{ 6-Stunden-Lauf } \\
\hline 1998 & 50.2 \\
\hline 1999 & 53.1 \\
\hline \multicolumn{2}{|c|}{ 12-Stunden-Lauf } \\
\hline 1992 & 75.9 \\
\hline 1995 & 84.8 \\
\hline \multicolumn{2}{|c|}{ 24-Stunden-Lauf } \\
\hline 1994 & 144.5 \\
\hline 1995 & 140.3 \\
\hline 1995 & 153.0 \\
\hline 1996 & 132.0 \\
\hline 1997 & 122.7 \\
\hline 1997 & 117.6 \\
\hline 1998 & 134.4 \\
\hline 1998 & 137.5 \\
\hline 1999 & 140.9 \\
\hline 1999 & 126.9 \\
\hline 2000 & 125.5 \\
\hline 2000 & 127.3 \\
\hline 2001 & 116.7 \\
\hline 2002 & 141.8 \\
\hline 2002 & 128.4 \\
\hline 2002 & 115.1 \\
\hline 2003 & 142.6 \\
\hline 2003 & 135.8 \\
\hline 2004 & 153.1 \\
\hline 2004 & 132.8 \\
\hline 2005 & 129.5 \\
\hline 2005 & 115.6 \\
\hline 2006 & 121.1 \\
\hline 2006 & 125.2 \\
\hline 2006 & 139.2 \\
\hline 2007 & 145.4 \\
\hline 2007 & 138.7 \\
\hline 2008 & 128.0 \\
\hline 2008 & 142.4 \\
\hline 2009 & 110.5 \\
\hline 2009 & 129.7 \\
\hline 2010 & 140.7 \\
\hline 2010 & 149.0 \\
\hline 2011 & 137.7 \\
\hline 2011 & 126.5 \\
\hline 2011 & 131.4 \\
\hline 2012 & 145.5 \\
\hline 2012 & 127.9 \\
\hline 2013 & 131.7 \\
\hline 2013 & 138.8 \\
\hline 2014 & 134.0 \\
\hline 2014 & 137.7 \\
\hline 2015 & 135.9 \\
\hline 2015 & 139.7 \\
\hline 2015 & 142.2 \\
\hline 2016 & 131.3 \\
\hline 2016 & 137.7 \\
\hline 2017 & 141.8 \\
\hline 2017 & 126.5 \\
\hline \multicolumn{2}{|c|}{ 48-Stunden-Lauf } \\
\hline 1999 & 181.8 \\
\hline 2000 & 208.2 \\
\hline 2001 & 170.6 \\
\hline 2002 & 183.5 \\
\hline 2003 & 178.2 \\
\hline 2005 & 186.7 \\
\hline \multicolumn{2}{|c|}{ 72-Stunden-Lauf } \\
\hline 2014 & 288.9 \\
\hline \multicolumn{2}{|c|}{ 6-Tage-Lauf } \\
\hline 2008 & 447.0 \\
\hline 2010 & 359.8 \\
\hline 2016 & 507.2 \\
\hline
\end{tabular}

523 Tabelle 2: Läufe aufgeteilt nach Dauer (6 Stunden bis 6 Tage) 
525

526

Abbildung 1

Verlauf des $\mathrm{HbA}_{1} \mathrm{c}$ über die Jahre

$527 \quad$ Abbildung 2

Verlauf der monatlichen Laufkilometer im Training über die

528

Jahre

529

Abbildung 3

Zusammenhang zwischen den $\mathrm{HbA}_{1} \mathrm{c}$-Werten und den

530

korrespondierenden Trainingslaufkilometern der 3 Monate vor

531

der jeweiligen $\mathrm{HbA}_{1} \mathrm{C}$-Messung

532

Abbildung 4

Verlauf der erzielten Kilometer in den 24-Stunden-Läufen über

533

die Jahre

534

535

536

537

538

539

540

541

542

543

544

545

546

547 


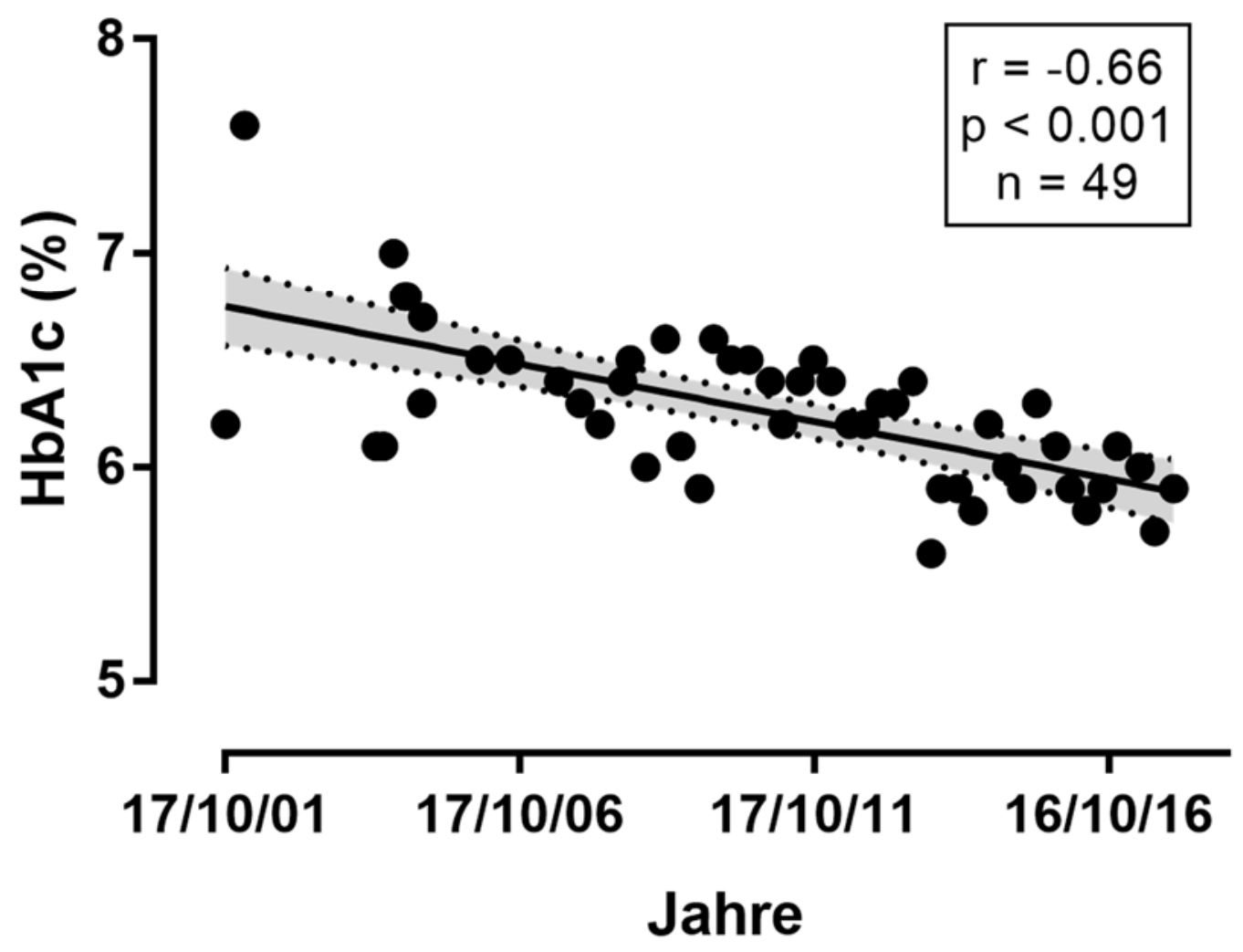

548

549 Abbildung 1

550

551 


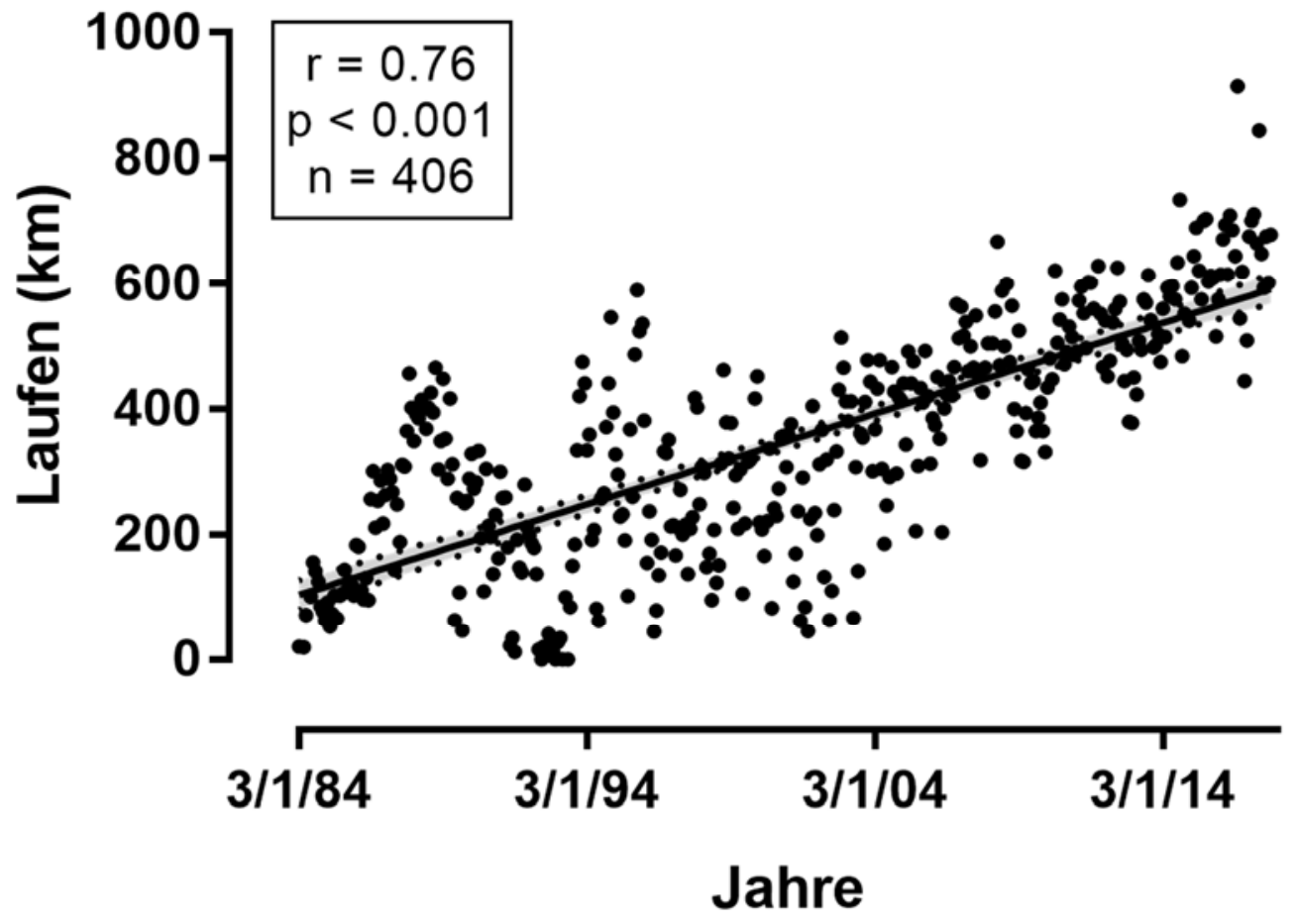

552

553 Abbildung 2

554

555

556

557

558

559

560

561

562

563

564 


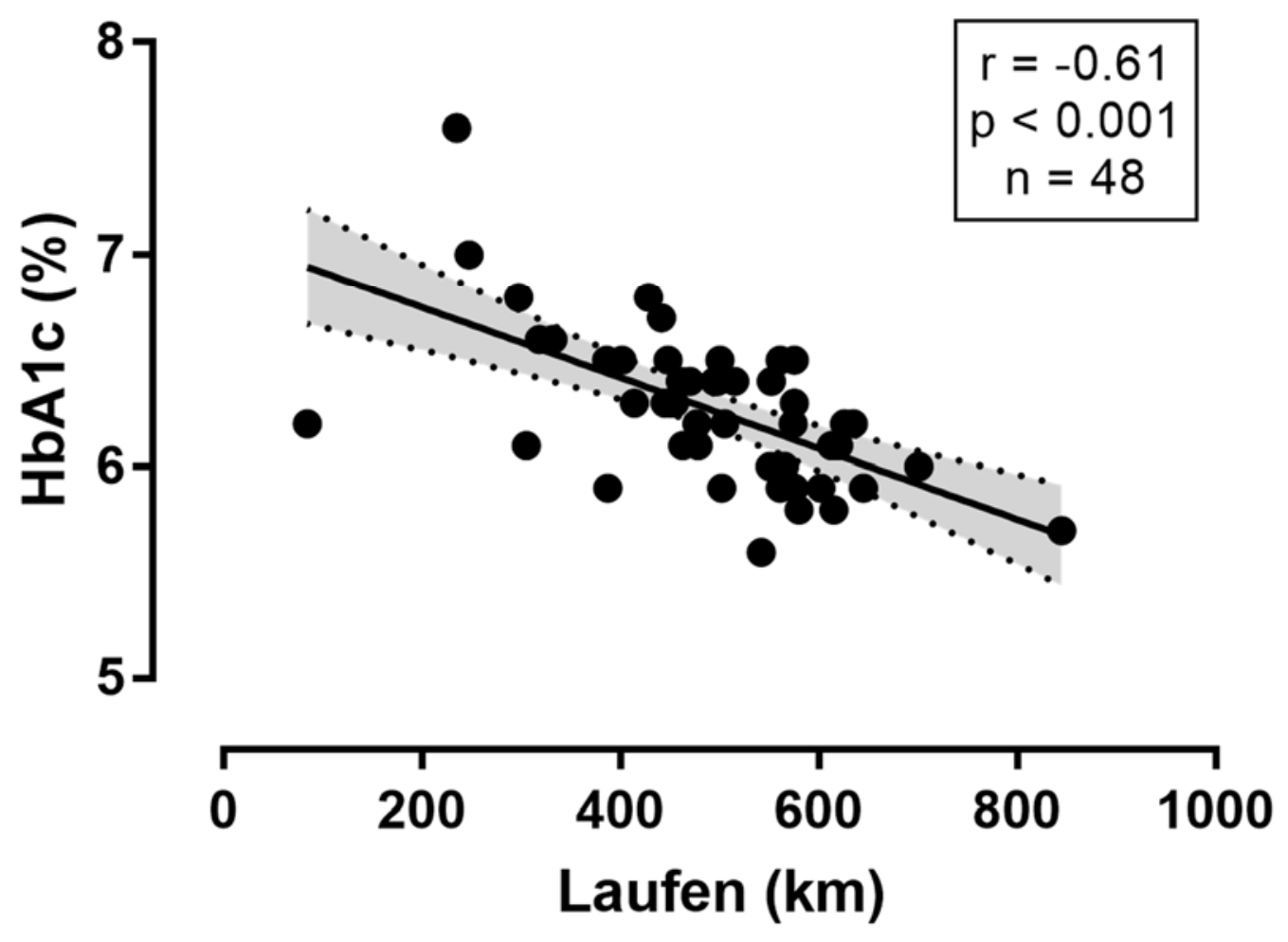

565

566 Abbildung 3

567

568

569

570

571

572

573

574

575 


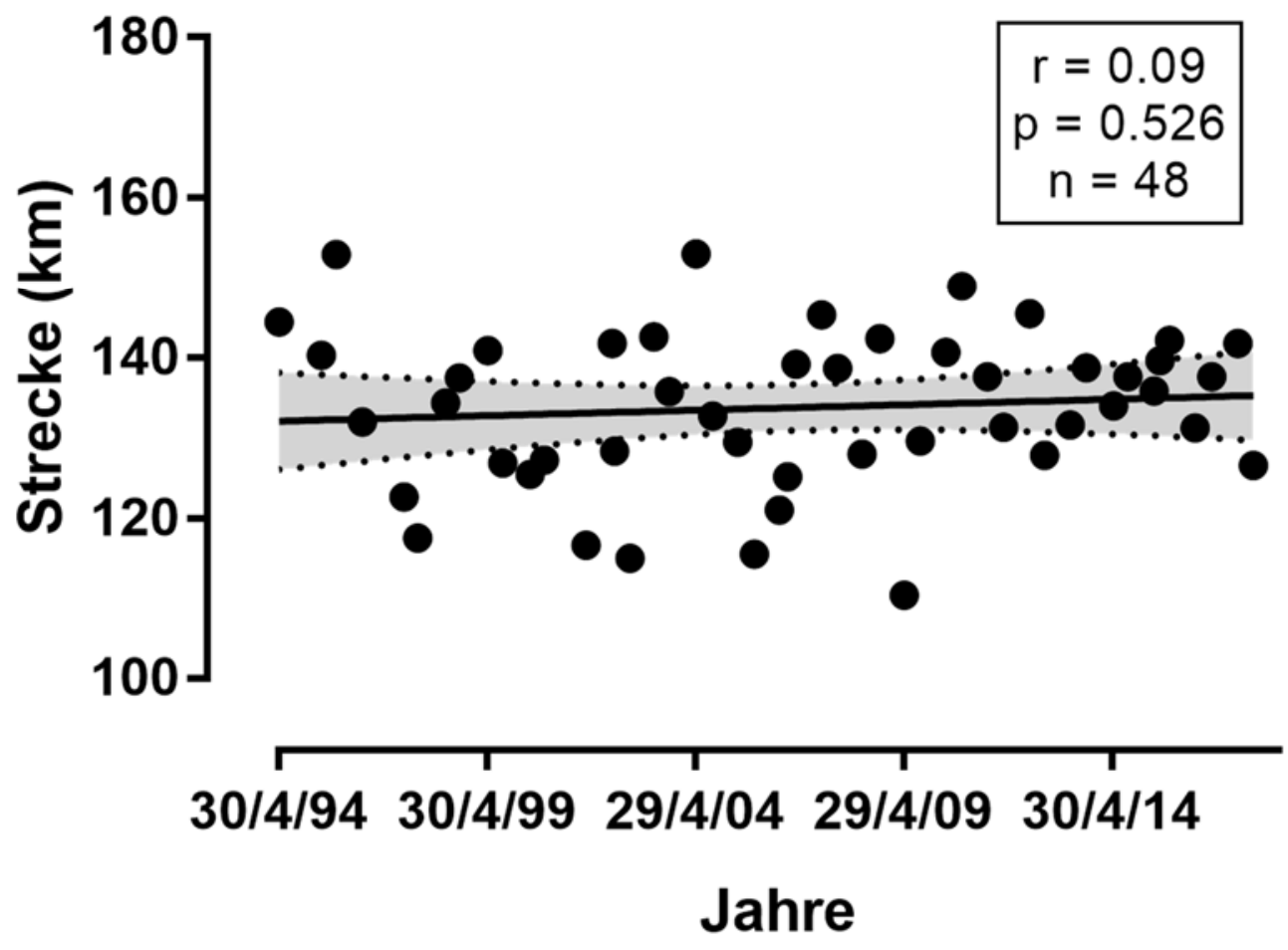

576

577 Abbildung 4

578 